\title{
VIEWPOINT
}

\section{Sleep disorders and gastrointestinal symptoms: chicken, egg or vicious cycle?}

\author{
M. MANEERATTANAPORN \& W. D. CHEY
}

Division of Gastroenterology, University of Michigan Health System, Ann Arbor, MI, USA

\section{INTRODUCTION}

Sleep disorders can arise as a consequence of a number of problems including difficulty falling or staying asleep, falling asleep at inappropriate times, excessive total sleep time, or abnormal behaviours associated with sleep. ${ }^{1}$ In Europe, the prevalence of sleep disorders amongst the general population has been estimated to range from $7 \%$ to $50 \% .^{2-5}$ Over 40 million Americans suffer from a sleep disorders. ${ }^{1}$ Sleep disorders have been found to slow response time, decrease attention and increase the likelihood of falling asleep at work. Moreover, sleep disorders have been found to affect adversely problem-solving skills and moral reasoning. ${ }^{6}$ Given these findings, it is not surprising that sleep disorders exert tangible effects on health, personal and public safety, productivity and quality of life. Recent evidence suggests that disturbed sleep is common amongst patients with gastrointestinal (GI) symptoms.

\section{PREVALENCE OF SLEEP DISORDERS IN GASTRO-OESOPHAGEAL REFLUX DISEASE AND IRRITABLE BOWEL SYNDROME}

Sleep disturbance is commonly reported by gastro-oesophageal reflux disease (GORD) sufferers. A telephone survey sponsored by the American Gastroenterological Association which queried 1000

Address for correspondence

Professor William D. Chey, MD, GI Physiology Laboratory, University of Michigan Health System, 3912 Taubman

Center, SPC 0362, Ann Arbor, MI 48109-0362, USA.

Tel: +1 734936 4775; fax: +1 734936 7392;

e-mail: wchey@umich.edu

Received: 11 December 2008

Accepted for publication: 30 December 2008 persons with at least weekly heartburn found that $79 \%$ of respondents experienced nighttime heartburn at least once per week. ${ }^{7}$ Of the patients with nighttime heartburn, $75 \%$ reported the effects on sleep and $40 \%$ felt that sleep difficulties impacted their ability to function the following day. Another recent survey from a large US community health plan found that over $80 \%$ of GORD sufferers experienced residual nighttime symptoms despite daily proton pump inhibitor (PPI) therapy. Approximately, $50 \%$ of patients with nighttime symptoms reported disturbed sleep, the most common problems being 'waking up at night' $(66.2 \%)$, 'trouble falling asleep again after waking up' $(52.6 \%)$ and 'trouble falling asleep' $(37.2 \%)^{8}$ It is important to note that the majority of the available data address the prevalence and clinical impact of disturbed sleep in patients with nighttime GORD symptoms. There are little data which have addressed the association between sleep disturbance and daytime GORD symptoms.

An association between sleep disturbance and irritable bowel syndrome (IBS) symptoms also appears to be emerging. Older studies have yielded conflicting results ${ }^{9-11}$ However, more recent studies support this association. ${ }^{12-14} \mathrm{~A}$ recent survey found that sleep disturbance was an independent predictor for the presence of IBS amongst nurses working different shifts at the University of Michigan. ${ }^{13}$ The study published by Cremonini et al. in this issue of Neurogastroenterology and Motility also lends evidence to support this association. The authors found a significant correlation between sleep disturbance and overall GI symptoms as measured by a validated survey. More specifically, they found that waking up at least four times a month was associated with a variety of GI symptoms including pain, urgency and diarrhoea. These finding were found to 
be independent of gender, age, body mass index and lifestyle factors such as smoking, alcohol consumption and physical activity. In aggregate, there seems little doubt of an association between sleep disturbance and GI symptoms. The question that remains is whether sleep disturbance is the cause or consequence of GI disorders such as GORD and IBS.

\section{SLEEP DISORDERS: CAUSATION OR CONSEQUENCE?}

Persistent GORD symptoms despite PPI therapy can arise as a consequence of ongoing acid or non-acid reflux or may be entirely unrelated to reflux. Patients are particularly susceptible to ongoing reflux while asleep as protective mechanisms such as the production and swallowing of bicarbonate rich saliva, oesophageal clearance mechanisms, and upper airway protective mechanisms are impaired..$^{15}$ In fact, recent data have linked more severe nighttime acid reflux as measured by $\mathrm{pH}$ monitoring, to poorer sleep quality. However, persistent acid or non-acid reflux can be demonstrated in only a subset of patients with residual symptoms despite PPI therapy. ${ }^{16}$ Further, even when reflux is present, the majority of reflux episodes are not sensed by patients. ${ }^{17}$ As such, mechanistic explanations for persistent daytime and nighttime GORD symptoms despite PPI therapy must be expanded beyond reflux.

Of the factors proposed to contribute to the pathogenesis of functional GI symptoms, there is intriguing evidence to support a link between disturbed sleep and the development of visceral hypersensitivity. In healthy volunteers, Onen et al. ${ }^{18}$ have shown that total sleep deprivation leads to hyperalgesia in response to painful mechanical stimulation applied to the thumb. They also observed an analgesic effect of 'recovery sleep'. Another group recently reported that more modest reductions in sleep time, as opposed to total sleep deprivation, induced somatic hyperalgesia as measured by finger withdrawal latency following heat stimulation. ${ }^{19}$ These authors also found that the loss of rapid eye movement (REM) sleep, in particular, led to the development of hyperalgesia. Cumulative loss of non-REM sleep over the course of 2 days eventually led to the levels of hyperalgesia observed after the more acute loss of REM sleep. Though such studies support an association between sleep loss and the development of somatic hyperalgesia, they may or may not be germane to the development of GI symptoms. A recent study by Schey et al. ${ }^{20}$ suggests that similar to somatic pain, sleep loss may well also adversely affect visceral sensation. In this crossover study which included 10 healthy volunteers and 10 patients with erosive oesophagitis, the investigators evaluated oesophageal sensation to acid perfusion following 3 days of sleep deprivation ( $<3 \mathrm{~h}$ per night) or 3 days of sufficient sleep ( $\geq 7 \mathrm{~h}$ per night). After sleep deprivation, GORD patients demonstrated significant changes in oesophageal sensation as measured by lag time to symptom report, symptom intensity rating and intensity score following oesophageal acid perfusion. Control subjects experienced no difference in oesophageal sensation after sleep deprivation or sufficient sleep, though trends favouring increased sensation after sleep deprivation were noted. Similar studies in IBS patients have thus far not been performed.

The mechanistic explanations for the link between sleep deprivation and hyperalgesia have not been well elucidated. Some of the factors proposed to play a role include disturbances in autonomic function, changes

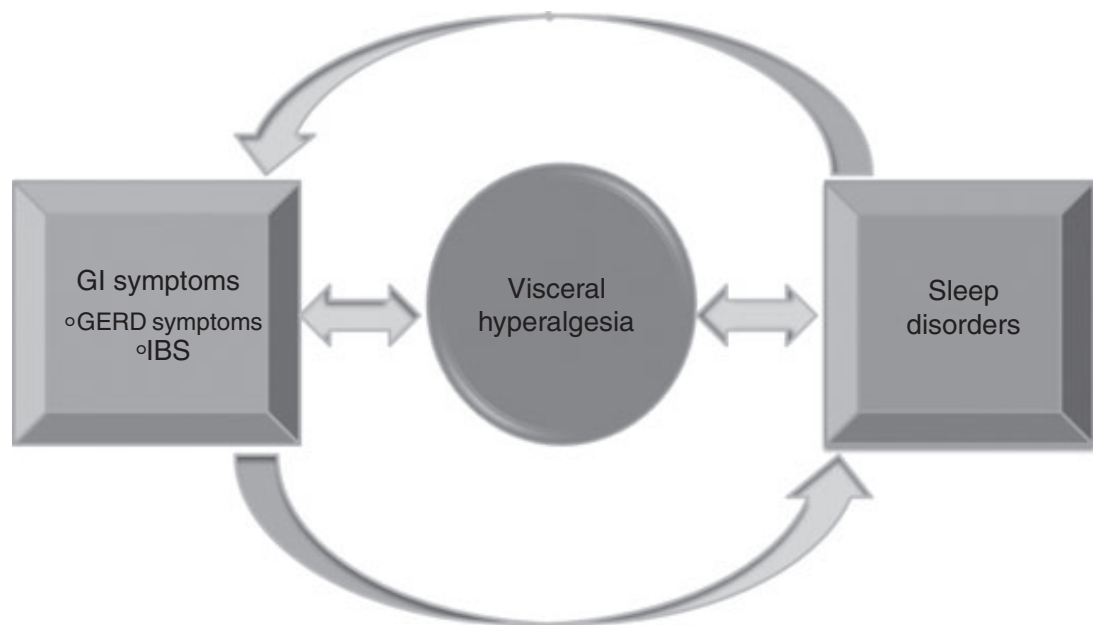

Figure 1 Proposed relationship between sleep disorders and gastrointestinal (GI) symptoms. 
in the concentration of neurotransmitters such as acetylcholine, adenosine, gamma aminobutyric acid, melatonin, and serotonin or amino acids such as glutamate, and alterations in the production and receptor binding of endogenous opiates. ${ }^{18,19}$ It is also possible that medications which affect REM sleep could play a role. For example, opiate analgesics including morphine and methadone are known to suppress REM sleep. ${ }^{21}$

\section{CONCLUDING COMMENTS}

Evidence supporting an association between disturbed sleep and GI symptoms continues to mount. It remains unclear as to whether sleep disturbance represents the 'chicken' or the 'egg' when it comes to GORD symptoms or IBS. It is likely that in this case, the chicken and the egg are not mutually exclusive. In other words, it seems reasonable to hypothesize that GI symptoms can in and of themselves disturb sleep. Primary or consequent disturbances in sleep can lead to changes in physiological parameters such as visceral sensation. This in turn worsens the severity and/or frequency of GI symptoms resulting in further problems with sleep. In this way, GI symptoms and disturbed sleep may well feed the development of a vicious cycle (Fig. 1). Many questions regarding the relationship between sleep and GI symptoms remain. For example, it will be important to document whether changes in visceral sensation following sleep deprivation occur in IBS patients. It will also be important to investigate whether restoration of normal sleep can positively impact upon visceral sensation and more importantly, GI symptoms. It is particularly interesting to envision the use of sleep deprivation as an experimental model for visceral hypersensitivity. It is hoped that unravelling the pathophysiology of the association between sleep and GI symptoms can move patients from counting sheep to sawing logs.

\section{REFERENCES}

1 Johnson DA. Gastroesophageal reflux disease and sleep disorders: a wake-up call for physicians and their patients. Rev Gastroenterol Disord 2005; 5(Suppl. 2): S3-11.

2 Fetveit A, Straand J, Bjorvatn B. Sleep disturbances in an arctic population: the Tromso Study. BMC Health Serv Res 2008; 8: 117.

3 Ohayon MM, Paiva T. Global sleep dissatisfaction for the assessment of insomnia severity in the general population of Portugal. Sleep Med 2005; 6: 435-41.

4 Ohayon MM, Partinen M. Insomnia and global sleep dissatisfaction in Finland. J Sleep Res 2002; 11: 339-46.
5 Punjabi NM, Bandeen-Roche K, Young T. Predictors of objective sleep tendency in the general population. Sleep 2003; 26: 678-83.

6 Balkin TJ, Rupp T, Picchioni D, Wesensten NJ. Sleep loss and sleepiness: current issues. Chest 2008; 134: 653-60.

7 Shaker R, Castell DO, Schoenfeld PS, Spechler SJ. Nighttime heartburn is an under-appreciated clinical problem that impacts sleep and daytime function: the results of a Gallup survey conducted on behalf of the American Gastroenterological Association. Am J Gastroenterol 2003; 98: 1487-93.

8 Chey WD, Mody R, Chen L et al. Nighttime symptoms and sleep impairment among patients with GERD receiving prescription proton pump inhibitors. Gastroenterology 2008; 134: A323-4.

9 Goldsmith G, Levin JS. Effect of sleep quality on symptoms of irritable bowel syndrome. Dig Dis Sci 1993; 38: 1809-14.

10 Jarrett M, Heitkemper M, Cain KC, Burr RL, Hertig V. Sleep disturbance influences gastrointestinal symptoms in women with irritable bowel syndrome. Dig Dis Sci 2000; 45: 952-9.

11 Fass R, Fullerton S, Tung S, Mayer EA. Sleep disturbances in clinic patients with functional bowel disorders. Am I Gastroenterol 2000; 95: 1195-2000.

12 Wei Zhen Lu, Gwee KA, Ho KY. Functional bowel disorders in rotating shift nurses may be related to sleep disturbances. Eur I Gastroenterol Hepatol 2006; 18: 623-7.

13 Nojkov B, Hoogerwerf S, Chey WD. The effect of shift work on the prevalence and clinical impact of functional bowel disorders in nurses. Am J Gastroenterol 2008; 103: S469.

14 Cremonini F, Camilleri M, Zinsmeister AR et al. Sleep disturbances are linked to both upper and lower GI symptoms in the general population. Neurogastroenterol Motil 2009; 21: 128-35.

15 Orr WC. Sleep and gastroesophageal reflux: what are the risks? Am J Med 2003; 115(Suppl. 3A): 109S-13S.

16 Mainie I, Tutuian R, Shay $\mathrm{S}$ et al. Acid and non-acid reflux in patients with persistent symptoms despite acid suppressive therapy: a multicentre study using combined ambulatory impedance-pH monitoring. Gut 2006; 55: 1398-402.

17 Tutuian R, Vela MF, Hill EG, Mainie I, Agrawal A, Castell DO. Characteristics of symptomatic reflux episodes on Acid suppressive therapy. Am J Gastroenterol 2008; 103: 1090-6.

18 Onen SH, Alloui A, Gross A, Eschallier A, Dubray C. The effects of total sleep deprivation, selective sleep interruption and sleep recovery on pain tolerance thresholds in healthy subjects. J Sleep Res 2001; 10: 35-42.

19 Roehrs T, Hyde M, Blaisdell B, Greenwald M, Roth T. Sleep loss and REM sleep loss are hyperalgesic. Sleep 2006; 29: 145-51.

20 Schey R, Dickman R, Parthasarathy S et al. Sleep deprivation is hyperalgesic in patients with gastroesophageal reflux disease. Gastroenterology 2007; 133: 1787-95.

21 Shaw IR, Lavigne G, Mayer P, Choiniere M. Acute intravenous administration of morphine perturbs sleep architecture in healthy pain-free young adults: a preliminary study. Sleep 2005; 28: 677-82. 